\title{
3 Research Square \\ Two Novel AMHR2 Gene Variants in Monozygotic Twins with Persistent Müllerian Duct Syndrome
}

\section{Hong Chen}

Fuzhou Children's Hospital of Fujian Medical University: Fuzhou Children's Hospital of Fujian Province

Peng Lin

Fuzhou Children's Hospital of Fujian Medical University: Fuzhou Children's Hospital of Fujian Province

Ruimin Chen ( $\square$ chenrm321@163.com)

Fuzhou Children's Hospital of Fujian Medical University https://orcid.org/0000-0003-4114-7706

\section{Yuan Xin}

Fuzhou Children's Hospital of Fujian Medical University: Fuzhou Children's Hospital of Fujian Province

\section{Research}

Keywords: Persistent müllerian duct syndrome, AMHR2 gene, Variants, AMH

Posted Date: August 3rd, 2021

DOl: https://doi.org/10.21203/rs.3.rs-728396/v1

License: (9) This work is licensed under a Creative Commons Attribution 4.0 International License.

Read Full License 


\section{Abstract}

Background: Persistent müllerian duct syndrome (PMDS) is an autosomal recessive congenital abnormality for which müllerian derivatives, uterus, cervix, upper 2/3 vagina and fallopian tubes, persist in an otherwise normally virilized males. Mutations in the $A M H$ gene and $A M H R 2$ gene have been identified as causative. However, there has been no functional experimental analysis of AMHR2 or AMH variants that caused PMDS. We ascertained a Chinese PMDS pedigree. To elucidate the pathogenic mechanisms of variant, we used complementation-based NanoLuciferase Binary Technology (NanoBiT) to examine $A M H$ and AMHR2 variants interaction in vivo. Using an ID-1 luciferase assay, we probed the effect of the two variants on the transcriptional activity of the TGF $\beta / B M P$ pathway.

Results: Molecular studies revealed two novel variants [c.118G>C/p. Gly40Arg, c.1222G>C/p. Ala408Pro] in the AMHR2 gene of our monozygotic boys. The AMHR2 p. Ala408Pro variants affect a functional domain, result in impaired substrate recognition and affinity. For p.Gly40Arg variant, the arginine residue will disfigure the local backbone and alter function. The AMHR2 p.Gly40Arg variant reduces its ability to bind to $\mathrm{AMH}$, while the p.Ala408Pro variant alters the kinase domain structure.

Conclusion: Both variants significantly reduce TGF $\beta / B M P$ signaling. This study provides new methods and concepts as to the study of the molecular pathogenic mechanism of PMDS.

\section{Introduction}

Persistent müllerian duct syndrome (PMDS) is a rare autosomal recessive inherited disorder of internal male sexual development, characterized by impaired regression of the müllerian ducts. To date, approximately 200 patients with PMDS have been reported since the initial description of this disease by Nilson[1].

The twin baby boys had 46, XY karyotype with external male genitalia, and the urethra meatus was at the tip of the penis (no hypospadias)[2]. However, both had internal müllerian duct structures, including a uterus, cervix, fallopian tubes, and upper two-thirds of the vagina. As reported, $45 \%$ of cases are caused by absolute or relative deficiencies of an anti-müllerian hormone (AMH), $40 \%$ due to $\mathrm{AMH}$ receptor type II (AMHR2), and yet in 15\%, the etiology is unknown [3]. In the male embryo, müllerian structures regress under the effect of $\mathrm{AMH}$ around the seventh week of gestation, releasing the testes from their initial positions in the pelvis to descend to the scrotum provided the cord is of sufficient length. If the müllerian ducts fail to regress, the testes remain suspended in the broad ligament. PMDS is usually diagnosed during orchidopexy or inguinal herniorrhaphy [3].

In this study, we describe the clinical characteristics of a pair of monozygotic twins with PMDS. Gene sequencing revealed two missense variants, c. $118 \mathrm{G}>\mathrm{C} / \mathrm{p}$.(Gly40Arg) and c. $1222 \mathrm{G}>\mathrm{C} / \mathrm{p}$.(Ala408Pro), in the $A M H R 2$ gene. We performed a series of functional assays to elucidate the pathogenic mechanisms underlying $A M H R 2$ variants. 


\section{Methods}

\subsection{Study subjects}

This study was reviewed and approved by the Ethics Committee of Fuzhou Children's Hospital of Fujian and was conducted in agreement with the Declaration of Helsinki Principles (NO. 201921). The twins were born to unrelated Chinese parents of Han ethnicity. Both lived in Fujian Province. Written informed consent was obtained from their parents.

\subsection{Targeted next-generation sequencing and data analysis}

Peripheral venous blood $(5 \mathrm{~mL})$ was collected from the twins and their parents for gene analysis. The extracted DNA was fragmented with DNAase, followed by PCR expansion and ligating the linker sequence, captured and purified twice by TruSight One Sequencing Panel (Illumina Inc, USA), and then amplified and purified by PCR. The final library was obtained in MiSeq sequencer (Illumina Inc, USA) which analyzed the exon region of 4811 for clinically relevant genes. The PMDS related genes were finally sequenced (http://www.illumina.com/).

All data were compared with the BWA algorithm to the reference sequence (UCSC hg19). The clinical data and bioinformatics software analyzed the results. The function, variation, and genetic pattern of each gene were inspected for suspicious candidate variants.

\subsection{Conservative and pathogenicity analysis of the variant}

We download various AMHR2 protein sequences through Uniprot (https://www.uniprot.org/) and the ClustalX program (http://ftp-igbmc.u-strasbg.fr/pub/ClustalX/) was used for sequence alignment. Sequence alignment results were displayed online using ConSurf (https://consurf.tau.ac.il/) [4]. The variants' pathogenicity was analyzed using the webserver PREDICT-SNP (https://loschmidt.chemi.muni.cz/predictsnp1/)[5].

\subsection{Three-dimensional structure modeling}

The amino acid sequence of wild-type human AMHR2 was retrieved from the UniProt database (http://www.uniprot.org). The 3D structure of the wild-type human AHMR2 using SWISS-MODEL (https://swissmodel.expasy.org/)[6; 7]. The online server Dynamut (http://biosig.unimelb.edu.au/dynamut/) was used to assess the local conformation of wild-type and variant proteins and assess changes in protein flexibility[8]. The structural representation was generated using the molecular visualization system in the open-source foundation PyMOL 2.4 (https://pymol.org/2/).

\subsection{Analysis of interaction between AMHR2 and AMH}

A structural complementation reporter system, NanoLuc $®$ Binary Technology (NanoBiT) (Promega, Cat\#N2014), analyzed protein interactions. NanoBiT consists of a Large BiT (LgBiT; 18kDa) subunit and a small complimentary peptide $(\mathrm{SmBiT} ; 3.6 \mathrm{kDa})[9]$. When the two target proteins interact, the LgBiT and 
SmBiT subunits converge to form an active enzyme and produce a bright luminescent signal in the presence of a substrate. The primers used for construction of plasmids are listed in Table S1. For NanoBiT protein interaction experiments, $A M H R 2$ gene $\mathrm{CDs}$ and the $\mathrm{N}$-terminus of the SmBiT were fused to form the AMHR2-SmBiT-N plasmid. The AMH gene and the C-terminus of LgBiT were fused to form the AMH-LgBiT-C expression plasmid. The wild-type full-length human AMHR2 (NM_001164690) and AMH (NM_000479) complementary DNA (cDNA) were chemically synthesized. HEK293T cells were cotransfected with AMHR2-SmBiT-N and AMH-LgBiT-C to assess the ability of the wild-type, p.Gly40Arg, and p.Ala408Pro of AMHR2 to interact with AMH. The cells were then transfected using jetOPTIMUS ${ }^{\circledR}$ in vitro DNA transfection reagent (Polyplus, Cat\#117-07). The DNA ratio was 1:1 when each different variant was co-transfected. Twenty-four hours after transfection, the culture medium was replaced with fresh Opti-

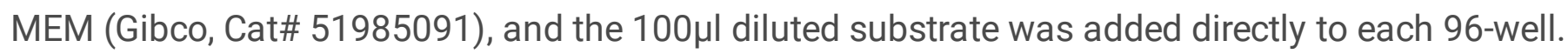
Transfection efficiency was determined by qPCR. Primers used for qPCR are listed in Table S2. The fluorescence intensity was measured with a Multimode Plate Reader VICTORNivo (Thermo Fisher, ND1000).

\subsection{TGF- $\beta / B M P$ luciferase assay}

For transient transfection, HEK-293T cells were then transfected using jetOPTIMUS $\AA$ in vitro DNA transfection reagent. HEK-293T cells were co-transfected with TGF- $\beta$ /BMP flash reporter plasmids, pGL4[luc2P/hID1/Hygro] (Promega, USA) wild-type or AMHR2 variants expression vectors. The DNA ratio of $A M H R 2$ and flash reporter plasmids were 1:1 when each different variant was co-transfected. Human recombinant AMH protein $(50 \mathrm{ng} / \mathrm{mL}$ ) (Sinobiological, CAT\# $17500-\mathrm{H} 07 \mathrm{H}$ ) was added according to the literature[10]. The Luciferase Reporter Assay System (Promega, USA) was used 48h after transfection.

\subsection{Cell culture}

Cell cultures were prepared and maintained according to standard cell culture procedures. The HEK293T cells was cultured in HG-DMEM (Invitrogen, USA) containing 10\% Fetal bovine serum (Gibco, USA).

\section{Results}

\subsection{Clinical evaluation}

\section{Case one}

A monozygotic 20-month-old Chinese male was referred to the pediatric surgery department of Fuzhou Children's Hospital of Fujian due to a left inguinal hernia and impalpable right testis. His height $(85.2 \mathrm{~cm})$ and weight $(9.8 \mathrm{~kg})$ were normal with male external genitalia and a penile length of $4.5 \mathrm{~cm}$ (normal for age on penile curve). The urethral opening was at the tip of the penis. The right testicle was not palpable in the scrotum or inguinal region. A left inguinal hernia was present. Ultrasonic examination detected a left testis in the groin and suspicious testis sonographic in the right iliac fossa. His serum AMH level was normal $(112 \mathrm{ng} / \mathrm{mL}$; normal $74.1 \sim 148.1 \mathrm{ng} / \mathrm{mL})$. Other relevant biochemical data are displayed in Table 1. Cytogenetic study in 50 metaphases revealed a 46, XY karyotype. Two weeks later, he underwent 
laparoscopic exploration for the gonad, high ligation of the hernia sac, and reduction of the testis. An ovarian-like tissue, fallopian tubes, and $1.5 \mathrm{~cm} \times 0.8 \mathrm{~cm}$ uterus was found intra-operative, but no apparent right testis. The left inguinal hernia was repaired, and ovarian-like tissue, fallopian tubes, uterus were extirpated. On pathology, the gonadal tissue was immature testicle with immature leydig and sertoli cells, and germ cells were absent(Figure 1A). Postoperative diagnosis: PMDS.

Table 1

Clinical evaluation data of the monozygotic twins

\begin{tabular}{|c|c|c|c|}
\hline \multirow[t]{2}{*}{ Items } & \multicolumn{2}{|l|}{ Patients } & \\
\hline & Twin one (II-1) & Twin two (II-2) & \\
\hline Age at initial diagnosis & 20-month-old & 20-month-old & \\
\hline Chromosome karyotype & $46, X Y$ & $46, X Y$ & \\
\hline Genitalia phenotype & $\begin{array}{l}\text { Impalpable right } \\
\text { testis, } \\
\text { left inguinal hernia }\end{array}$ & $\begin{array}{l}\text { Bilateral cryptorchidism, } \\
\text { transverse testicular } \\
\text { ectopia }\end{array}$ & \\
\hline $\begin{array}{l}\text { Blood hormonal } \\
\text { characteristics }\end{array}$ & & & Normal value \\
\hline $\mathrm{FSH}$ & 0.16 & 0.13 & $0.00 \sim 5.50 \mathrm{IU} / \mathrm{L}$ \\
\hline LH & 0.77 & 0.80 & $0.00 \sim 4.10 \mathrm{IU} / \mathrm{L}$ \\
\hline DHT & 17.34 & 85.96 & $\mathrm{pg} / \mathrm{ml}$ \\
\hline Androstenedione & $\mathbb{\nabla 0 . 3}$ & $\bigotimes 0.3$ & $0.7 \sim 3.6 \mathrm{ng} / \mathrm{ml}$ \\
\hline DHEA-S & $\otimes 15$ & \$15 & $80 \sim 560 \mathrm{ug} / \mathrm{dl}$ \\
\hline Testosterone & 2.5 & 2.5 & $\mathrm{ng} / \mathrm{ml}$ \\
\hline $\begin{array}{l}\text { Testosterone of post-hCG } \\
\text { level }\end{array}$ & - & 373.7 & $\mathrm{ng} / \mathrm{ml}$ \\
\hline $\mathrm{AMH}$ & 108.0 & 112.0 & $\begin{array}{l}74.1 \sim 148.1 \\
\mathrm{ng} / \mathrm{mL}\end{array}$ \\
\hline Inhibin B & 163.85 & 139.48 & $\begin{array}{l}33.78 \bowtie 339.64 \\
\mathrm{pg} / \mathrm{ml}\end{array}$ \\
\hline
\end{tabular}

Case two

Considering that his twin was diagnosed with PMDS, he was subsequently examined in our hospital. Physical examination of the patient revealed male external genitalia with an empty scrotum, no palpable testes, and a penile length of $5 \mathrm{~cm}$ (normal for age). The urethral opening was at the tip of the penis. A 
$1.0 \mathrm{~cm} \times 0.8 \mathrm{~cm}$ mass was present in the right inguinal region, and the left testis was not found in the inguinal area. Ultrasound examination revealed both testes of standard size in the right inguinal canal. Serum AMH level was normal $(108 \mathrm{ng} / \mathrm{mL}$; normal $74.1 \sim 148.1 \mathrm{ng} / \mathrm{mL})$. Other relevant biochemical data are displayed in Table 1. Karyotype analysis showed 46, XY. By sonographic image, the prostate could be detected. During exploratory surgery, bilateral testes were found in the right groin, and a uterus and bilateral fallopian tubes were found in the pelvic cavity (Fig. 1B). Postoperative diagnosis: PMDS. Detailed clinical characteristics of the monozygotic twins are presented in Table 1.

\subsection{Genetic diagnosis}

Mutational analysis of the AMHR2 gene identified pathogenic mutations in the monozygotic twins witch confirmed the diagnosis of PMDS. Two novel variants, c. $118 \mathrm{G}>\mathrm{C} / \mathrm{p}$. (Gly40Arg) and c. $1222 \mathrm{G}>\mathrm{C} / \mathrm{p}$. (Ala408Pro), of the $A M H R 2$ gene were identified in this case. The father carried the heterozygous mutation c. $1222 \mathrm{G}>\mathrm{C} /$ p.(Ala408Pro). Whereas, the mother carried the heterozygous mutation c.118G > C/p.(Gly40Arg) (Fig. 2A, 2B). The two variants were not present in the $1000 \mathrm{G}$ and ExAC databases.

To further elucidate the genetic testing results, we analyzed the interspecific conservation and pathogenicity of this variant. We compared AMHR2 protein sequences in 13 mammals, including Homo sapiens, Pan troglodytes, Macaca mulatta, Myotis lucifugus, Equus caballus, Ailuropoda melanoleuca, Felis catus, Canis lupus familiaris, Bos taurus, Sus scrofa, Oryctolagus cuniculus, Loxodonta africana, and Mus musculus. Therefore, the residues at positions 40 and 408 of AMHR2 are highly conserved across these representative mammals (Fig. 2C). Both missense variants of $A M H R 2$ were predicted to be deleterious for all six predictors (PredictSNP, MAPP, PhD-SNP, PolyPhen1, PolyPhen2, and SIFT), deducing a deleterious effect of both variants with a high confidence score (Table 2). Hence, both variants, c.118G $>$ C/p.(Gly40Arg) and c. $1222 \mathrm{G}>\mathrm{C} / \mathrm{p}$.(Ala408Pro), in AMHR2 are almost certaining pathogenic.

Table 2

Predictions and confidence scores for the AMHR2 variants obtained with the PREDICT-SNP server

\begin{tabular}{|lll|}
\hline Variants & $\begin{array}{l}\text { p.Gly40Arg } \\
\text { (Prediction; expected accuracy) }\end{array}$ & $\begin{array}{l}\text { p.Ala408Pro } \\
\text { (Prediction; expected accuracy) }\end{array}$ \\
\hline PredictSNP & Deleterious; 72\% & Deleterious; 87\% \\
\hline MAPP & Deleterious; 46\% & Deleterious; 77\% \\
\hline PHD-SNP & Deleterious; 58\% & Deleterious; 82\% \\
\hline PolyPhen-1 & Deleterious; 74\% & Deleterious; 74\% \\
\hline PolyPhen-2 & Deleterious; 81\% & Deleterious; 63\% \\
\hline SIFT & Deleterious; 53\% & Deleterious; 43\% \\
\hline
\end{tabular}

\subsection{Three-dimensional structure modeling}


To further examine the structural changes in AMHR2, we used the SWISS-MODEL online software to conduct modeling of the protein structure (Fig. 3A, 3D). The DynaMut web server analyzed and visualize protein dynamics and assessed the impact of these variants. Local interactions between wild-type and variant amino acid residues were probed via the DynaMut online server. Give the change difference between glycine (Gly) and Arginine (Arg), the variant Gly40Arg introduces an aberrant change leading to the repulsion of ligands or other residues with similar charge. The torsion angles at Gly 40 for this residue are deviant insofar as only glycine is flexible enough to create these torsion angles. The arginine residue will compel the local backbone into an abnormal confirmation and disturb the local structure (Fig. 3B, 3C). The variant p.Ala408Pro causes a significantly different between alanine (Ala) and proline (Pro). In addition, position 408 of the amino acid sequence of AMHR2 is located within the protein kinase domain, the substitution of Ala408 by a proline, known to break a-helices, possibly hindering this functional domain. Also, proline causes hydrogen bonding to be lost and introduces hydrophobic bonds, which may disturb with the correct folding of the protein (Fig. 3E, 3F).

\subsection{The affinity between AMHR2 variants and $A M H$}

We employed a luciferase-based protein fragment complementation assay to characterize the interaction of AMHR2 and AMH (NanoBiT). A clear luminescent signal was obtained with this experimental method when the SmBit-AMHR2-N and LgBit-AMH-C were co-expressed, indicating a strong interaction with this combination (Fig. 4A, 4B). Relative AMHR2 mRNA expression levels were analyzed 24h post-transduction by qPCR. There was no difference in the AMHR2 levels (Figure S1). The data shown are RLU. The wildtype values were set at an arbitrary mean value of 1 , and the fold change in the mutant group was calculated based on the wild-type group. As shown in Fig. 4C, the binding ability of the p.Gly40Arg variant of AMHR2 to AMH was significantly decreased to $32.3 \sim 49.2 \%$ of the wild-type. As expected, the p.Ala408Pro variant did not impact the affinity with $\mathrm{AMH}$.

\subsection{TGF- $\beta / B M P$ luciferase assay}

To determine the effect of the variant on the AMH/AMHR2 signal pathway, we evaluated the transcriptional activity of TGF- $\beta$ /BMP by DNA-Binding protein inhibitor ID-1 promoter-luciferase assay. As expected, the luciferase activity, as driven by the ID-1 transcriptional response elements, had increased significantly when HEK-293T cells were transfected with ID-1 promoter flash reporter plasmids and wildtype or variant expression vectors (especially in the wild-type group). Data shown were relative luminescence units (RLU) (Fig. 4D). The wild-type values were designated at an arbitrary mean value of 1 , and the fold change in the mutant group was calculated according. At 48 hours after transfection, the variants of either the p.Gly40Arg or p.Ala408Pro significantly diminished the signaling pathway of AMH/AMHR2 to $29.0 \%$ and $5.1 \%$ ofthe wild-type, respectively.

\section{Discussion}


PMDS is a sui generis inherited disorder of sexual differentiation characterized by failure of regression of the müllerian ducts in $\mathrm{XY}$ males[2]. We studied a Chinese family with this rare disorder having pathogenic mutations in AMHR2. Compound heterozygous mutations were uncovered in monozygotic twins and heterozygous. The clinical phenotype of $A M H$ and $A M H R 2$ mutations are characteristically indistinguishable[11]. Typically, a male reproductive tract and a male phenotype are present with inconspicuous fallopian tubes and uterine structures. Approximately $55 \%$ of patients have bilateral cryptorchidism, $20 \%$ unilateral cryptorchidism and/or contralateral hernia, and a small patient have an uterine inguinal hernia. Before nine months of age, reducing the testis may prevent deterioration of reproductive function[12]. Transverse testicular ectopia (TTE) is an uncommon congenital male reproductive dysplasia in which both testicles are found in the same inguinal canal[13; 14]. Approximately $25 \sim 30 \%$ of patients with PMDS also have TTE[15; 16; 17]. TTE is associated with PMDS, yet the role of $\mathrm{AMH}$ in the process of testicular descent is unclear, possibly related to gubernaculum testis function[18]. Recent studies have shown that AMH may shorten the gubernendar line in humans[19]. It is plausible that the irregular adhesion between the fetal testis and the müllerian remnants beget TTE. Whereas, our monozygotic boys harboring the had the same genotype had disparate different phenotypes. One had an inguinal hernia and unilateral cryptorchidism, and the sib had TTE. The genotype of $A M H$ and $A M H R 2$ genes are independent of phenotype[11;20;21;22; 23]. Environmental or other genetic factors may lead to discordant phenotypes despite identical genotypes, but a specific mechanism is unclear. Given that the testes are the sole source of $\mathrm{AMH}$, serum AMH levels reflect functional Sertoli cells. Serum AMH is depressed in patients with $A M H$ gene variants, whereas high or average concentrations are typical of $A M H R 2$ variants. Thus, normal $\mathrm{AMH}$ levels usually exclude $\mathrm{AMH}$ synthesis mutations. On the other hand, AMH variant p.(GIn496His), hinders ligand binding[24]. Bear in mind that normal serum AMH is not necessarily an $A M H R 2$ gene variant because in $15 \%$ of patients a genetic cause is not identified. PMDS diagnosis requires differentiation from dysgenetic male pseudohermaphroditism and mixed gonadal dysgenesis (MGD). The dysgenetic male pseudohermaphroditism results from the complete failure of both testes. With the persistence of müllerian duct structures along with the ambiguity of external genitalia, MGD is characterized by a contralateral streak gonad, typically in the setting of $45, \mathrm{X} / 46, \mathrm{XY}$, or $46, \mathrm{XY}$ karyotype. Müllerian duct structures are preserved along with the ambiguity of external genitalia.

AMHR2 gene is located in 12q13, including 11 exons, encoding three functional receptor areas: the extracellular domain (exon $1 \sim 3$ ), a single transmembrane hydrophobic region (exon 4), an intracellular serine/threonine kinase domain (exon 5 11). According to the Human Gene Mutation Database (HGMD Professional, 2020.4), about 103 different deleterious AMHR2 gene variants have been identified in which give rise to PMDS. The kinase domain (amino acid 203-518) serves a vital role in identifying the substrate. Position 408 of the amino acid sequence of AMHR2 is located within the protein kinase domain and the substitution of Ala408 by a proline, known to break a-helices, could disrupt interfering with this domain and interfere with the correct folding of the protein. In our case,the AMHR2 p. Ala408Pro variants affect a functional domain, result in impaired substrate recognition and affinity. For p.Gly40Arg variant, the arginine residue will disfigure the local backbone and alter function. The unique structural 
disturbance of p.Gly40Arg variant modifies the local hydrogen bond pattern of AMHR2, resulting in decreased variant flexibility. Known protein-protein interactions require the flexibility of local domains to ensure protein function and activity[25;26]. That is to say, the Gly40Arg variant deform the structure of the extracellular domain and hanpers ligand binding. To date, meticulous and effective methods to evaluate the interaction between AMHR2 variants and AMH are lacking in previous studies. We evaluated the effect of the binding ability of the AMHR2 variants to its ligand AMH by a luciferase-based protein fragment complementation assay. Our results revealed that the binding ability of p.Gly40Arg variant to AMH was profoundly reduced. As mentioned earlier, the flexibility of the extracellular domain of AMHR2 mutant protein is compromised, which may disturb AMH binding. Finally, using the TGF- $\beta$ /BMP by DNABinding protein inhibitor ID-1 promoter-luciferase assay, we demonstrated that both variants significantly attenuated the transduction of the AMH/AMHR2 signaling pathway. Taken together, we have assessed the change in AMH binding ability of the AMHR2 variants by a new approach, and demonstrate the pathogenicity of both variants identified in our twins.

\section{Conclusions}

To sum, monozygotic twins with PMDS are presented with two novel variants in the AMHR2 gene. Our results showed that the p.Gly40Arg variant of AMHR2 had a reduced ability to bind to $A M H$, while p.Ala408Pro variant undermines the function of the kinase domain, both of which synergistically diminish AMH/AMHR2 signaling. Our findings advance insight into the clinical evaluations and molecular basis of PMDS.

\section{Abbreviations}

AMH: anti-müllerian hormone; AMHR2: anti-müllerian hormone receptor type II; MGD: mixed gonadal dysgenesis; PMDS: Persistent müllerian duct syndrome; TTE: Transverse testicular ectopia.

\section{Declarations}

\section{Acknowledgments}

The authors are grateful to the family members and patients for their participation in this study.

\section{Authors' contributions}

Chen Hong conducted the data analysis and interpretation and wrote the manuscript. Peng Lin and Chen ruimin contributed to the study design and helped to analyze data and revise the first draft. Yuan Xin assisted in clinical case data collection. All authors read and approved the final manuscript.

\section{Funding support}


This work was sponsored by the grants from the Key Clinical Special Discipline Construction Program of Fuzhou, Fujian, P.R.C (No:201610191), Clinical medical center (201808310), The Basic and Clinical Study of Rare Disease (No: ZD-2019-01).

\section{Availability of data and materials}

The dataset supporting the conclusions of this article is included within the article.

\section{Ethics approval and consent to participate}

This study was approved by the Ethics Committee of Fuzhou Children's Hospital of Fujian Medical University, and written informed consents were obtained from the participants' legal guardians.

\section{Ethics approval and consent to participate}

This study was approved by the Ethics Committee of Fuzhou Children's Hospital of Fujian Medical University, and written informed consents were obtained from the participants' legal guardians.

\section{Consent for publication}

Not applicable.

\section{Conflict of interest}

Authors declare no conflicts of interest.

\section{References}

1ه Farikullah J, Ehtisham S, Nappo S, et al. Persistent Müllerian duct syndrome: lessons learned from managing a series of eight patients over a 10-year period and review of literature regarding malignant risk from the Müllerian remnants. BJU Int. 2012;110(11 Pt C):E1084-1089.

2هUnal E, Karakaya AA, Beştaş A, et al. Identification of four novel variant in the AMHR2 gene in six unrelated Turkish families. J Endocrinol Invest. 2021;44(6):1301-1307.

3\Pulido L, Iwasiuk G, Sparkuhl M, et al. Persistent Mullerian Duct Syndrome Presenting in an Incarcerated Recurrent Inguinal Hernia with Hydrocele. Urol Case Rep.2017;12:47-48.

4『Ashkenazy H, Abadi S, Martz E, Chay O, Mayrose I, Pupko T, and B.-T. N, ConSurf 2016: an improved methodology to estimate and visualize evolutionary conservation in macromolecules. Nucleic Acids Res.2016;44(W1):W344-350.

5खBendl J, Stourac J, Salanda O, et al. PredictSNP: robust and accurate consensus classifier for prediction of disease-related mutations. PLoS computational biology. 2014;10(1):e1003440. 
6囚Studer G, Rempfer C, Waterhouse AM, et al. QMEANDisCo-distance constraints applied on model quality estimation. Bioinformatics.2020;36(6):1765-1771.

$7 \llbracket$ Waterhouse A, Bertoni M, Bienert S, et al. SWISS-MODEL: homology modelling of protein structures and complexes. Nucleic Acids Res.2018;46(W1):W296-W303.

8\Rodrigues $\mathrm{CH}$, Pires $\mathrm{DE}$, Ascher DB. DynaMut: predicting the impact of mutations on protein conformation, flexibility and stability. Nucleic Acids Res. 2018;46(W1):W350-W355.

9『Dixon AS, Schwinn MK, Hall MP, et al. NanoLuc complementation reporter optimized for accurate measurement of protein interactions in cells. ACS chemical biology2016;11(2):400-408.

10खMalone SA, Papadakis GE, Messina A, et al. Defective AMH signaling disrupts GnRH neuron development and function and contributes to hypogonadotropic hypogonadism. Elife.2019;e47198.

11囚Hutson JM, Grover SR, O'Connell M, et al. Malformation syndromes associated with disorders of sex development. Nat Rev Endocrinol.2014;10(8):476-487.

12囚Mazen I, El-Gammal M, McElreavey K, et al. Novel AMH and AMHR2 Mutations in Two Egyptian Families with Persistent Müllerian Duct Syndrome. Sex Dev.2017;11(1):29-33.

13囚Yıldız A, Yiğiter M, Oral A, et al. Transverse testicular ectopia. Pediatr Int. 2014;56(1):102-105.

$14 \llbracket Y u$ J, Wang L, Ma S, et al. Detection and Treatment of Persistent Mullerian Duct Syndrome With Transverse Testicular Ectopia. Urology. 2020;140:e4-e5.

$15 \llbracket$ Shalaby MM, Kurkar A, Zarzour MA, et al. The management of the persistent Müllerian duct syndrome. Arab J Urol.2014;12(3):239-244.

16『Kaul A, Srivastava KN, Rehman SM, et al. Persistent Müllerian duct syndrome with transverse testicular ectopia presenting as an incarcerated inguinal hernia. . Hernia.2011;15(6):701-704.

17区Picard JY, Cate RL, Racine C, et al. The Persistent Müllerian Duct Syndrome: An Update Based Upon a Personal Experience of 157 Cases. Sex Dev.2017;11(3):109-125.

18区Alp BF, Demirer Z, Gürağaç A, et al. Persistent Mullerian duct syndrome with transverse testicular ectopia and seminoma. Int Urol Nephrol.2014;46(8):1557-1562.

19هHutson JM, Lopez-Marambio FA. The possible role of AMH in shortening the gubernacular cord in testicular descent: A reappraisal of the evidence. J Pediatr Surg. 2017;52(10):1656-1660.

20खLang-Muritano M, Biason-Lauber A, Gitzelmann C, et al. A novel mutation in the anti-müllerian hormone gene as cause of persistent müllerian duct syndrome. Eur J Pediatr.2001;160(11):652-654. 
21区Mazen I, Abdel Hamid MS, El-Gammal M, et al. AMH gene mutations in two Egyptian families with persistent müllerian duct syndrome. Sex Dev.2011;5(6):277-280.

22هMorikawa S, Moriya K, Ishizu K, et al. Two heterozygous mutations of the AMH gene in a Japanese patient with persistent Müllerian duct syndrome. J Pediatr Endocrinol Metab. 2014;27(11-12):1223-1226.

23『van der Zwan YG, Brüggenwirth HT, Drop SL, et al. A novel AMH missense mutation in a patient with persistent Müllerian duct syndrome. Sex Dev.2012; 6(6):279-283.

24囚Belville C, Van Vlijmen H, Ehrenfels C, et al. Mutations of the anti-mullerian hormone gene in patients with persistent mullerian duct syndrome: biosynthesis, secretion, and processing of the abnormal proteins and analysis using a three-dimensional model. Mol Endocrinol.2004;18(3):708-721.

25『Pauwels K, Tompa P. Editorial: Function and Flexibility: Friend or Foe? Front Mol Biosci. 2016;3:31.

26囚Jarosch R. The alpha-helix, an overlooked molecular motor. Protoplasma. 2005;227(1):37-46.

\section{Figures}

A

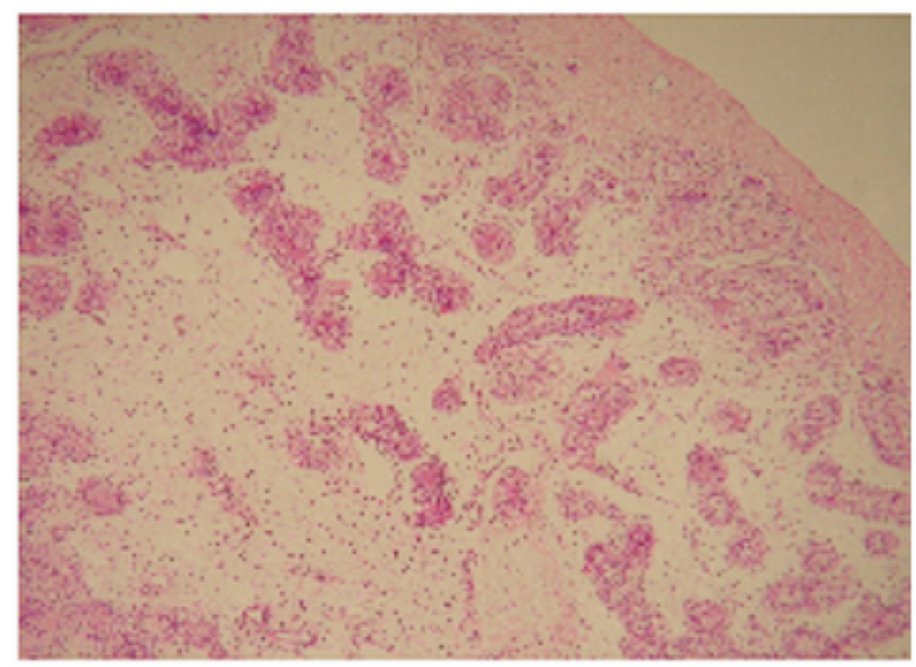

B

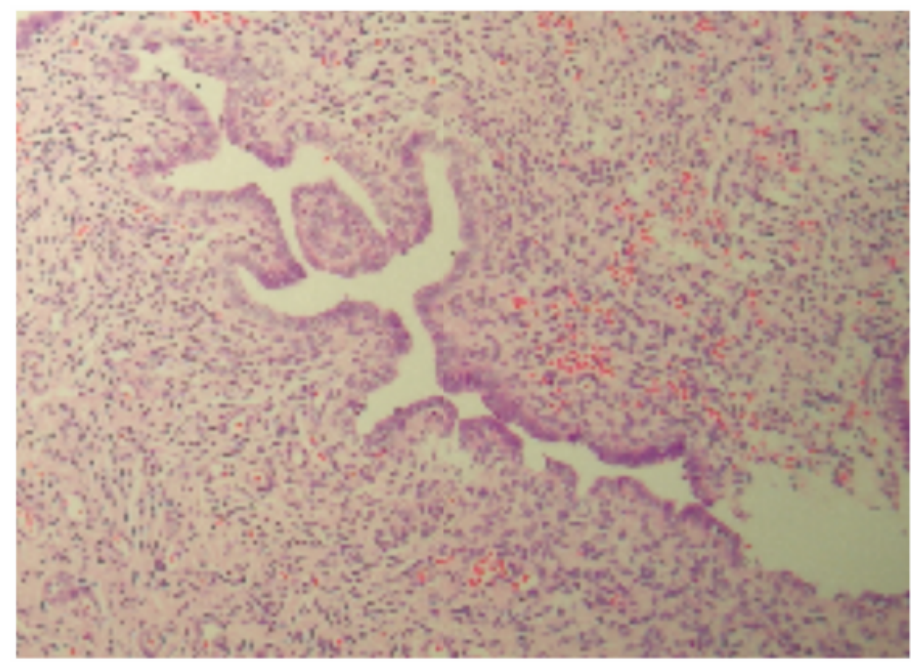

\section{Figure 1}

Pathologic examination. A: Pathologic examination of case one. Leydig and Sertoli cells were immature, and germ cells were absent. B: Pathologic examination of case two. Endometrium and endometrium stromal tissue. 
A

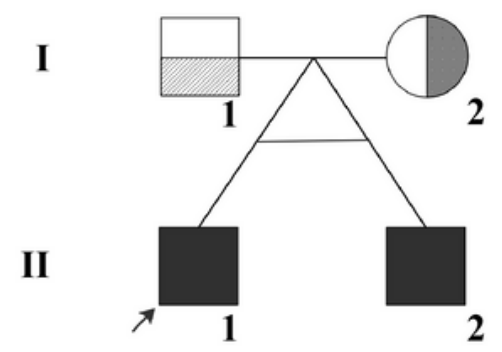

B

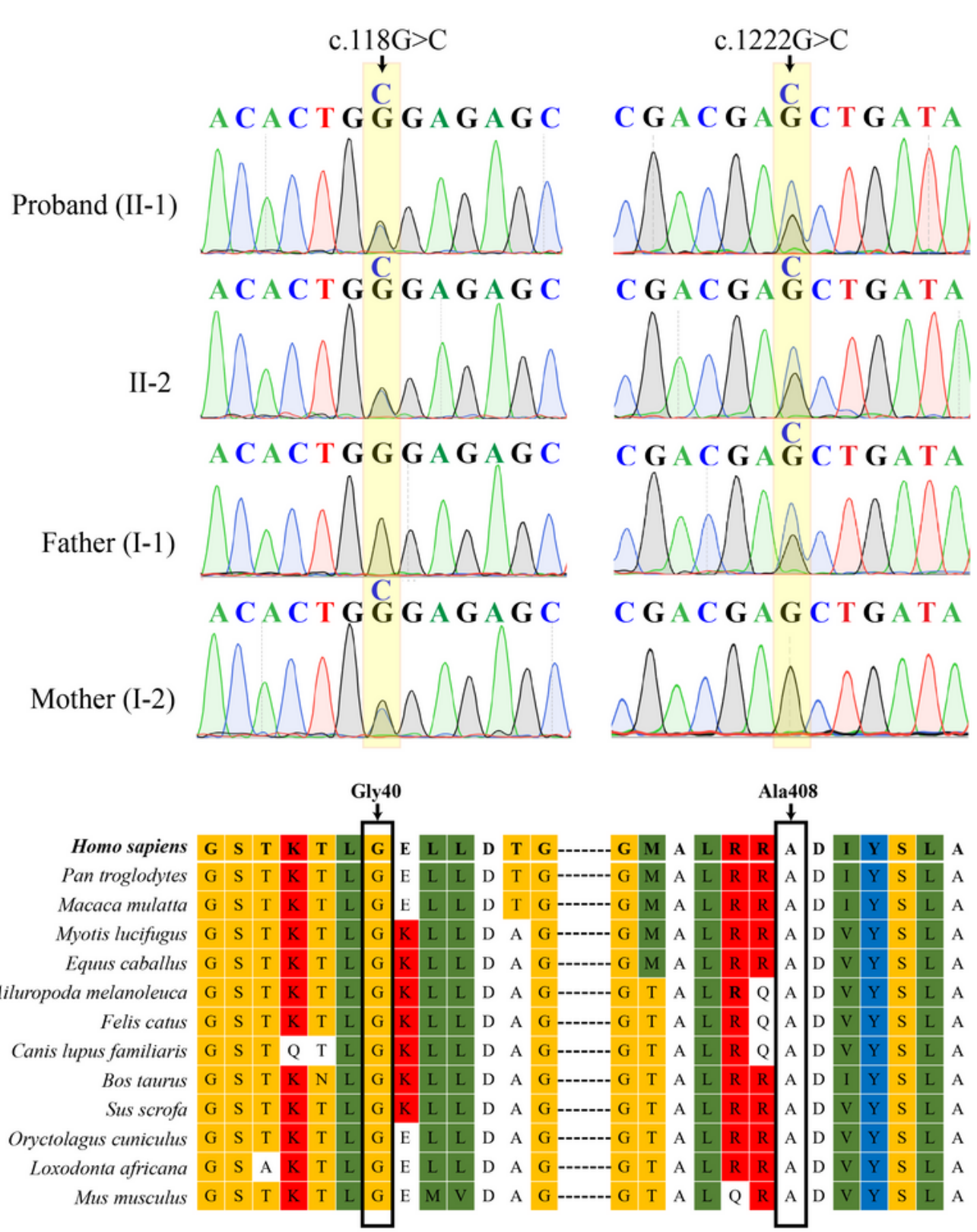

\section{Figure 2}

AMHR2 gene variants in the twins and their family members. A: The pedigree of the family with PMDS. Squares represent males and circles represent females. The proband is indicated by an arrow. Affected individuals are shown as filled black symbols, half-filled symbol is clinically unaffected subjects harboring a heterozygous variant. B: Sanger sequencing chromatograms showing that the twins carry the compound heterozygous variants [c.118G>C/p.Gly40Arg, c.1222G>C/p.Ala408Pro] in the AMHR2 gene. 
Mother ( $\mathrm{I}-2)$ has heterozygous c.118G $>\mathrm{C}$ variant, and ather $(\mathrm{I}-1)$ carries a the heterozygous $\mathrm{C} .1222 \mathrm{G}>\mathrm{C}$ variant. C: A conservative analysis across diverse mammals.

A

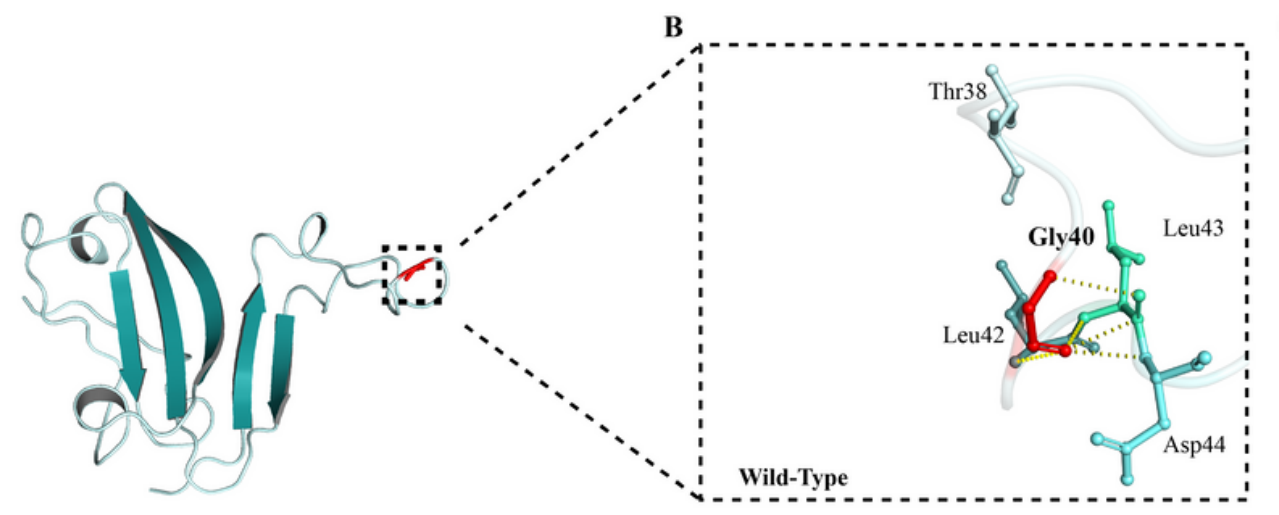

D

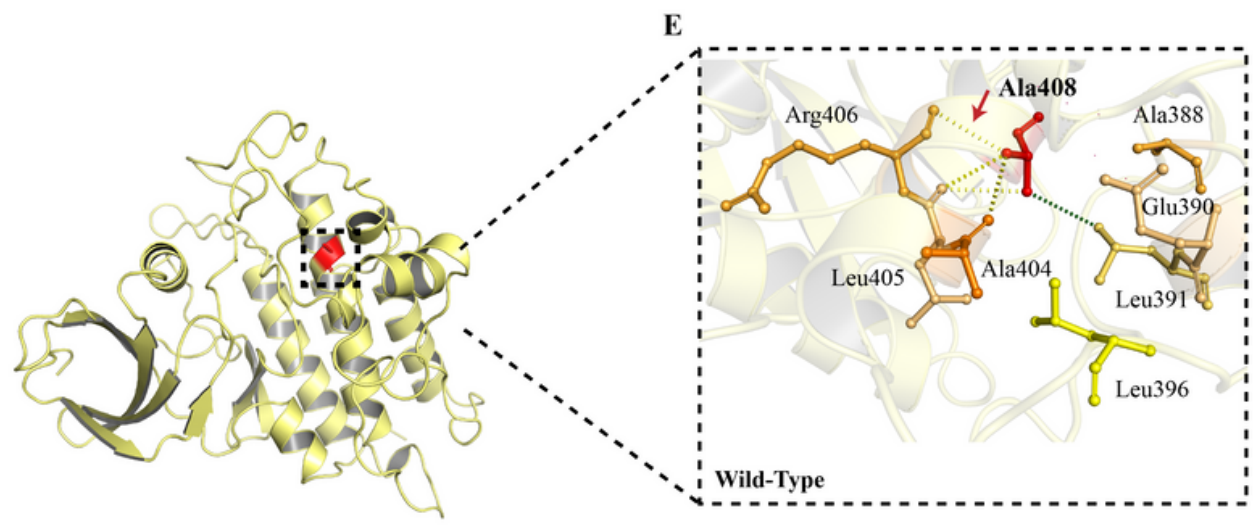

C

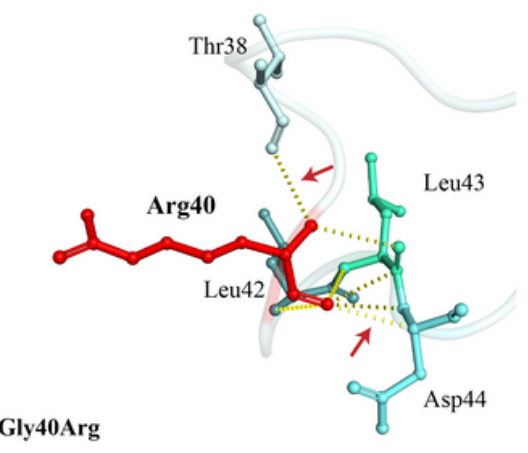

F

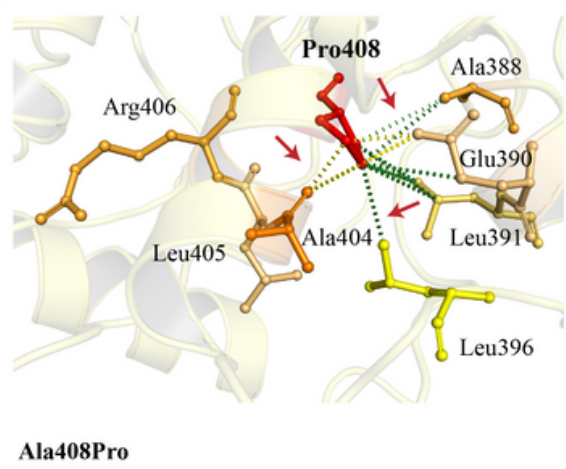

Figure 3

Prediction of interactions between amino acid residues. (A) The extracellular structure of AMHR2 protein. (B) Local structure of wild-type AMHR2 protein. (C) Local structure of AMHR2 p.Gly40Arg variant. (D) Schematic diagram of intracellular AMHR2 structure. (E) Local structure of wild-type AMHR2 protein. (F) Local structure of AMHR2 pp.Ala408Pro variant. Wild-type and variant residues are colored in red and are also represented as sticks alongside the surrounding residues which are involved in other types of interactions. The red arrows mark interactions between the altered amino acids, and the black arrows point to wild-type or variant amino acid residues. Hydrogen bonding is shown with a yellow dotted line. Hydrophobic bonds are indicated by a dotted green line. 
A

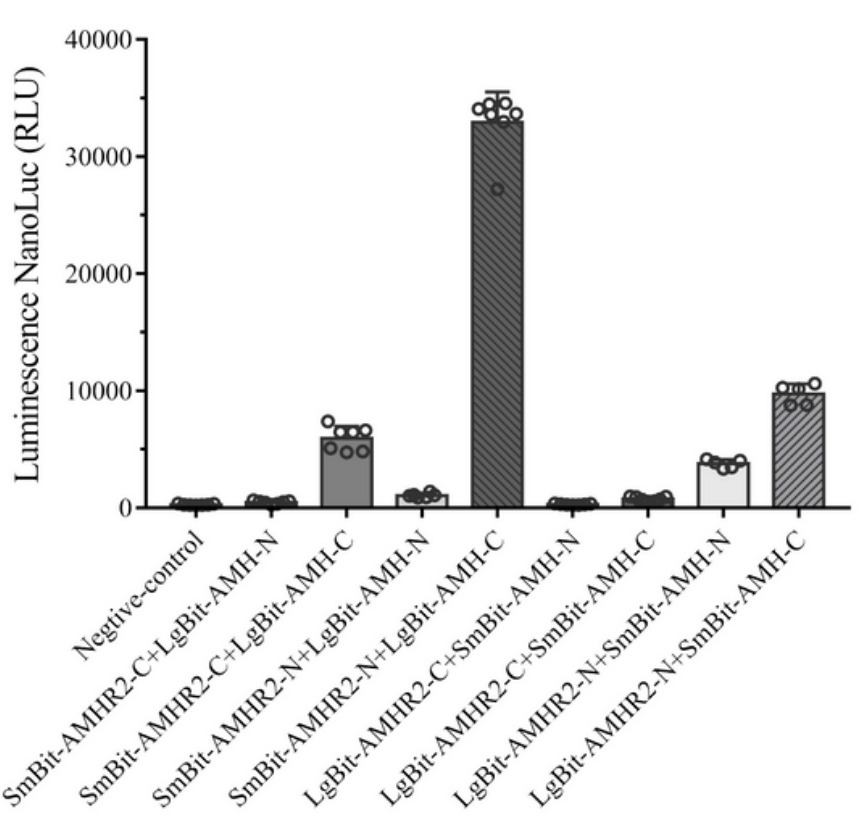

$\mathrm{C}$

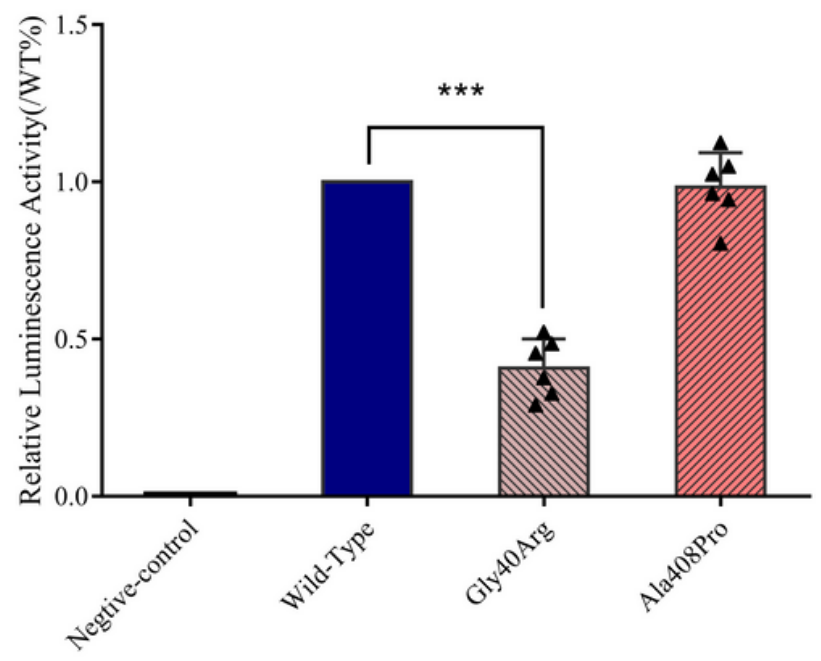

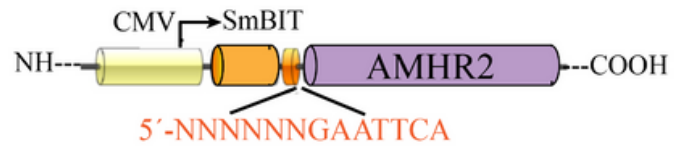
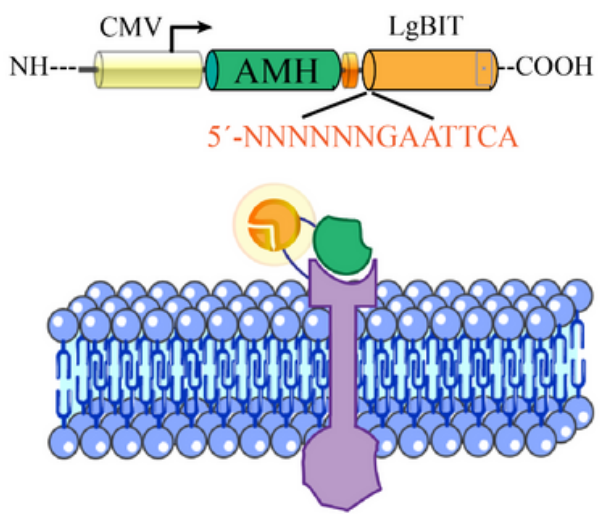

$\mathrm{D}$

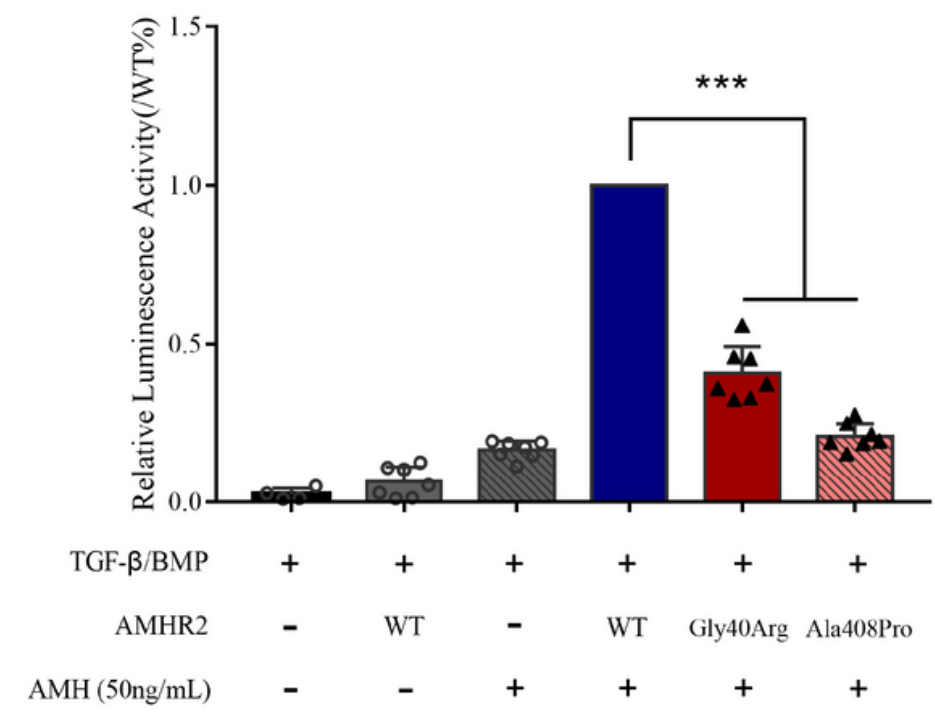

\section{Figure 4}

Functional analysis of AMHR2 variants. (A-C) The interaction between AMHR2 variants and AMH. (A) HEK293 cells were transiently transfected with eight different combinations of LgBit and SmBit fusion proteins to screen the fusion protein combinations for maximum response. (B) The AMHR2 gene and the $\mathrm{N}$-terminus of the SmBit was fused to form the AMHR2-SmBiT-N plasmid. The AMH gene and the Cterminus of LgBit were fused to form the AMH-LgBit-C expression plasmid. Schematic structure of the AMHR2 and AMH NanoBit-fused proteins. (C) Twenty-four hours after co-transfection, the diluted substrate was directly added to each well to measure the absolute value of NanoBit activity for 30 minutes. For comparison, the wild-type values were adjusted to 1', and the relative value of AMHR2-AMH binding capacity is represented by a histogram. (D) Co-transfected HEK293T cells with wild-type or AMHR2 variants expression plasmids and TGF- $\beta$ /BMP flash reporter plasmids. The plus $(+)$ and minus $(-)$ signs below the $X$-axis indicate whether TGF- $\beta$ /BMP flash reporter plasmids, AMHR2 and AMH, have 
been added, respectively. For comparison, the wild-type values was adjusted to 1 ', and the relative value of transcriptional activities is represented by a histogram. ${ }^{*} P<0.05$, ${ }^{\star *} P<0.01$ and ${ }^{*} * * P 0.001$.

\section{Supplementary Files}

This is a list of supplementary files associated with this preprint. Click to download.

- FigureS1.tif

- TableS1Primersforplasmidconstruction.docx

- TableS2PrimerlistforqPCR.docx 